\title{
Amyloid cystic lung disease presenting in a patient with human immunodeficiency virus
}

\begin{abstract}
A 30-year-old gentleman with a medical history of HIV, on HAART therapy and pulmonary amyloidosis proven by lung biopsy presented with progressive, worsening shortness of breath and chronic productive cough over the last year. Pulmonary function tests showed a severely obstructive process and CT scan of the thorax manifested diffusely thin-walled, air-filled cysts. After reviewing the patient's prior histories, PFTs and CTof the thorax, the most likely cause of his symptoms was amyloid-related cystic lung disease. After being placed on a LABA/ICS, and LAMA inhaler, the man's condition remained stable. Active oncologic and pulmonary surveillance were continued every three to six months as the patient's respiratory status, PFTs and imaging remained stable. Thoughts of chemotherapy and lung transplant have been suggested, but not initiated at this time.

Amyloid-related cystic lung disease is a rare presentation of amyloidosis in the thorax. It has been associated with collagen vascular diseases and mucosa-associated lymphoid tissue lymphoma. After an extensive literature search, it does not appear that this condition has been referenced in an HIV-positive patient; thus making this report unique. More case reports will need to be published and reviewed to see whether an association with HIV and amyloid-associated cystic lung disease exists.
\end{abstract}

Key words: amyloidoisis, amyloid cystic lung disease, HIV

Adv Respir Med. 2020; 89: 324-327

\section{Introduction}

Amyloidosis is a broad term used to characterize extracellular tissue deposition of plasma proteins in a pathological insoluble fibrillar form [1]. These deposits can occur in many different organs and therefore cause a constellation of clinical conditions. Amyloid-related cystic lung disease is one of the rarer conditions in the thorax. At this time, it has been associated with collagen vascular diseases (most commonly being Sjörgen Syndrome) and mucosa-associated lymphoid tissue lymphoma (MALToma) [2]. After an extensive literature search, it does not appear that amyloid-related cystic lung disease has been referenced in a human immunodeficiency virus (HIV)-positive patient.

\section{Case presentation}

A 30-year-old gentleman was referred to our pulmonary clinic in 2014 by his medical oncologist due to concerns of his worsening shortness of breath over the last year in the setting of pulmonary amyloidosis proven by surgical lung biopsy two years earlier (Figure 1). His medical history was also significant for HIV with recent initiation of highly active antiretroviral therapy (HAART) and a recent cluster of differentiation 4 (CD4) count of 300 . His HIV has been complicated by HIV-associated mucosa-associated lymphoid tissue (MALT) lymphoma status post chemotherapy and pneumocystic pneumonia, both in August 2013. He also had an 8-year history of smoking a pack per day but quit over a year earlier. 

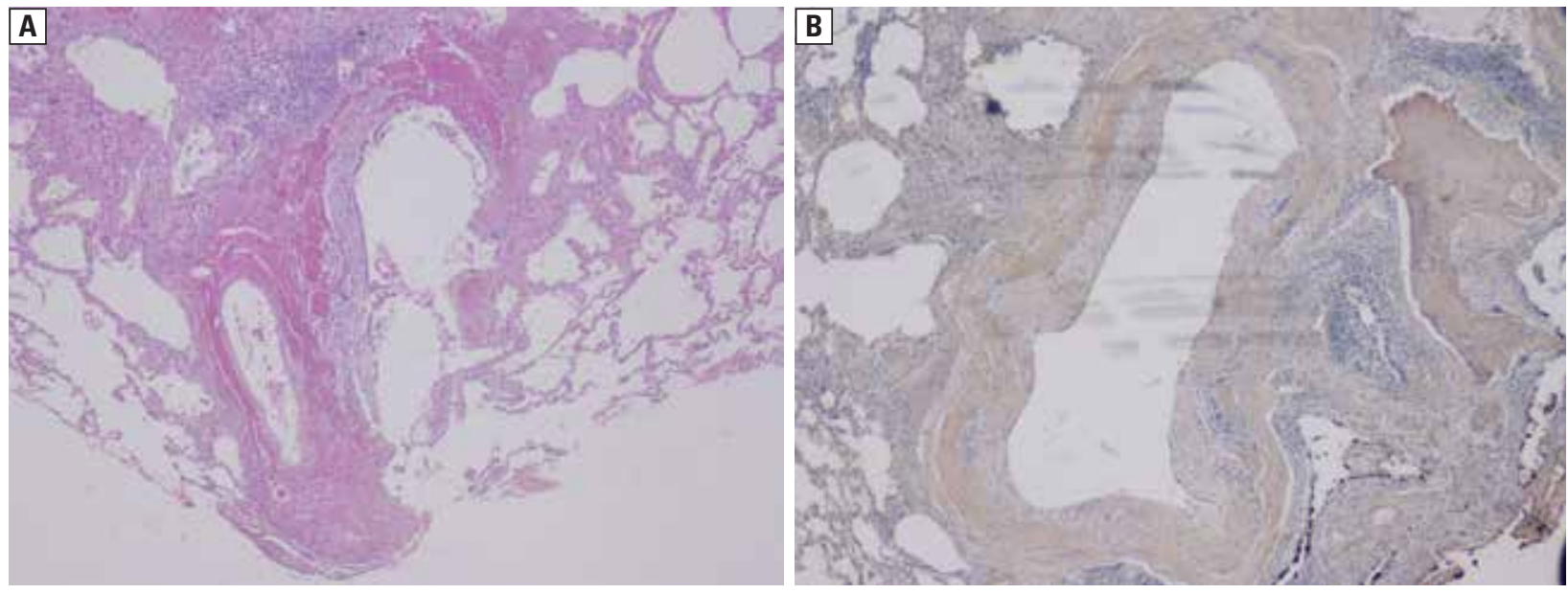

Figure 1. The section shows lung parenchyma with extensive pale pink, extracellular, glassy, hyaline material around the blood vessels. The congo red staining shows orange color of the amyloid deposits

This was the patient's first presentation to our pulmonary clinic. He described a history of a chronic cough that was productive in nature and worsening shortness of breath over the last year. The patient now finds he gets short of breath whenever he tries to minimally exert himself. At baseline, the patient qualified for $2 \mathrm{~L}$ of home oxygen, which he stated he wore throughout the night and usually around 4-5 hours during the day. He also noted an increased need for his albuterol inhaler, now requiring its use 4-5 times per week. The patient denied any orthopnea or paroxysmal nocturnal dyspnea with his shortness of breath. At the time of initial assessment in the clinic, the man was found to have normal vitals except mild tachypnea with a respiratory rate of 20; however, he had a peripheral oxygen saturation of $94 \%$ on room air. On physical exam he had a mild expiratory wheeze diffusely in both lung fields; otherwise, the rest of his physical examination was within normal limits.

The patient recently had pulmonary function tests (PFTs), which showed a severely obstructive process. The forced expiratory volume $1\left(\mathrm{FEV}_{1}\right)$ was $0.97 \mathrm{~L}$ ( $29 \%$ predicted), the forced expiratory volume 1 over forced vital capacity $\left(\mathrm{FEV}_{1} / \mathrm{FVC}\right)$ was $64 \%$ predicted, the total lung capacity (TLC) was $6.46 \mathrm{~L}$ (101\% predicted) with no improvement after bronchodilator, residual volume was $4.24 \mathrm{~L}$ (232\% predicted) and diffusion capacity of carbon monoxide (DLCO) was $15.2 \mathrm{~mL} / \mathrm{mm}$ $\mathrm{Hg} / \mathrm{min}$ (60\% predicted).

In addition, the man had a recent computed tomography (CT) scan of his thorax that showed diffusely distributed thin-walled, air-filled cystic spaces which had increased in number since his previous CT scan two years earlier. A majority of the cystic spaces appeared to be centrilobular in distribution; however, they were also noted extending to the pleural surfaces and paraseptal locations. Lung apices also appeared to be less affected than mid to lower lung segments. Scattered 2-3mm nodules were redemonstrated compared to previous CT scans; however, the number of nodules had decreased and were replaced by areas of cystic lung destruction (Figs 2, 3). There were no features consistent with bronchiolitis obliterans such as a mosaic pattern. A transthoracic echocardiogram (TTE) revealed an ejection fraction of 55 to $60 \%$ with normal diastolic function. No abnormalities of cardiac leaflets or chambers were noted; right ventricle was normal in size, thickness and function, but unfortunately, no mention of right ventricular systolic pressure (RVSP) was noted.

At this time, the working diagnosis was pulmonary amyloidosis in the setting of HIV and HIV-associated MALT lymphoma causing obstructive symptoms clinically manifesting as shortness of breath and productive cough. However, there were plans to check alpha-1 antitrypsin level as this is also high on the differential. Due to the patient's symptoms and evidence of wheezing on examination, the man had budesonide-formoterol fumarate added to his inhaled medication regimen. He continued to take his previously prescribed albuterol as needed. He was counseled to continue using his oxygen regularly, and he was referred to the Infectious Disease clinic for appropriate vaccinations. The patient was to follow up pulmonary checkup every three months or for worsening symptoms.

The man continued to follow up in the pulmonary clinic approximately every three months 


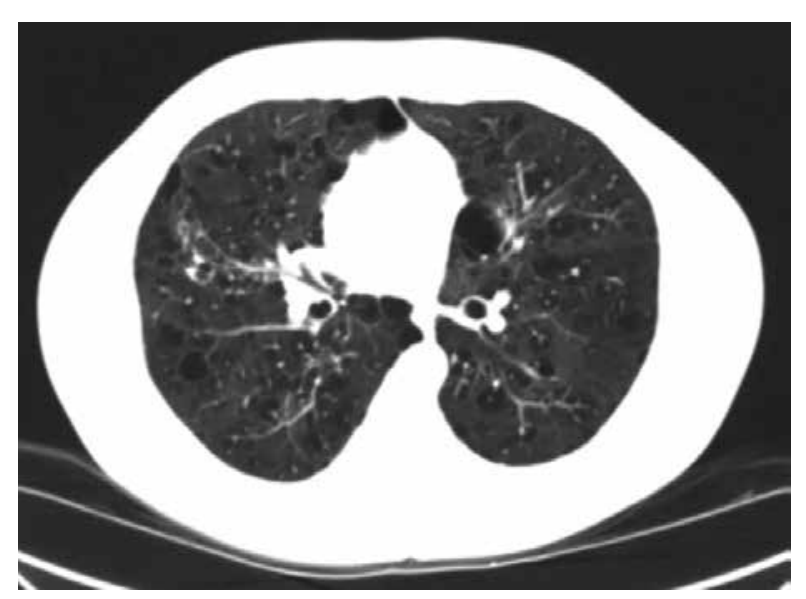

Figure 2. Axial chest CT image showing diffuse cystic lesions with scattered micronodules
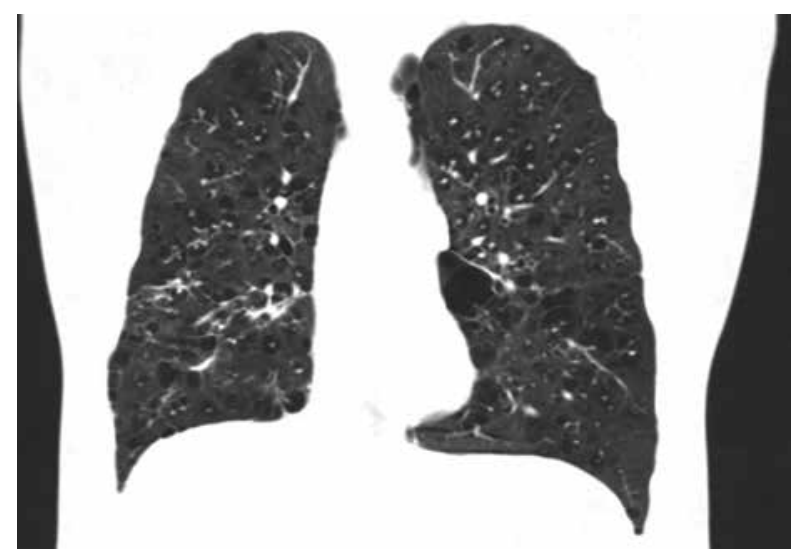

Figure 3. Coronal chest CT image showing diffuse cystic lesions with scattered micronodules

as requested. His alpha-1 antitrypsin level came back within normal limits. Despite adherence to the inhaler regimen of budesonide-formoterol fumarate used twice daily and albuterol as needed, the patient continued to have significant shortness of breath with minimal exertion and carried on with a productive cough. Due to poor symptom control, aclidinium bromide was also added to his regimen. Again, the man had minimal, if any, improvement with these two scheduled inhalers. Repeat PFTs were performed that showed a relatively stable severely obstructive process one year after initial visit with a $\mathrm{FEV}_{1}$ of $0.74 \mathrm{~L}$ from $0.97 \mathrm{~L}$ ( $29 \%$ to $22 \%$ predicted), a $\mathrm{FEV}_{1} / \mathrm{FVC}$ of $68 \%$ predicted (from $64 \%$ predicted), a TLC of 6.08 from 6.46 L (94\% from 101\% predicted) with no improvement after bronchodilator, residual volume increasing to 4.36 from $4.24 \mathrm{~L}$ (from $232 \%$ predicted to $230 \%$ predicted) and a DLCO of 15.9 from $15.2 \mathrm{~mL} / \mathrm{mm} \mathrm{Hg} / \mathrm{min}$ (from
$60 \%$ predicted to $57 \%$ ). At this time, a discussion was held with the patient's oncologist regarding the treatment of his pulmonary amyloidosis. The oncologist did not believe starting chemotherapy targeting plasma cells would help reverse his pulmonary status. Active oncologic and pulmonary surveillance were continued every three to six months as the patient's respiratory status and PFTs remained relatively stable. Referral for lung transplantation evaluation was discussed with the patient and he did not want to consider this option at this time.

\section{Discussion}

Amyloidosis is a generic term to describe a group of disorders associated with the pathological extracellular tissue deposition of plasma proteins in an insoluble fibrillar form. These deposits can occur in many different organs and therefore cause a constellation of clinical conditions. Amyloid light-chain (AL) is the most common type of amyloidosis associated with thoracic involvement [3]. Other noncardiac intrathoracic amyloid-related patterns that have been described are: tracheobronchial, parenchymal with nodular and diffuse alveolar septal subtypes, intrathoracic lymphadenopathy, parenchymal with diffuse alveolar septal subtype and pleural disease [4]. Amyloid-related cystic lung disease is one of the rarer conditions in the thorax.

The pathomechanism of cyst formation in amyloidosis is not fully understood; however, at this time, the studies indicate that pulmonary elastolysis via elastolytic matrix metalloproteinases is the common denominator between cystic lung light chain deposition disease and other cystic lung diseases. It is postulated that initially, there is recruitment of macrophages around light chain deposits, which cause elastin destruction via the production of matrix metalloproteinases. This in turn causes fragility of the lung parenchyma and enlargement and rupture of the affected alveoli and bronchioles [5].

Knowledge regarding this disease is limited at this time as only a few case reports are available for review. After surveying twenty-one cases, the most common pulmonary function pattern seen were normal (42\%) and obstructive (32\%) [2]. Imaging reports for these cases typically found cysts that were: multiple defined as greater than or equal to $10(67 \%)$, round $(100 \%)$ or lobulated $(95 \%)$; thin-walled defined as $<2 \mathrm{~mm}(81 \%)$, small defined as $<1 \mathrm{~cm}(100 \%)$ to moderate defined as $1-2 \mathrm{~cm}$ in size (81\%) [2]. The most commonly 
associated diseases were collagen vascular diseases (57\%) and the most common being Sjörgen Syndrome (10 of the twelve cases). Pulmonary mucosa-associated lymphoid tissue lymphoma (MALToma) were also diagnosed in 33\% of cases.

The major question then arises: how are we certain that the patient's symptoms were secondary to this rare disease rather than a plethora of more common etiologies? The man's differential diagnosis was very broad for his worsening shortness of breath. Emphysema could explain the patient's symptoms; however, he only had an eight pack-year smoking history, which is unlikely to explain the severity of his symptoms, the degree of obstruction seen on PFTs and the amount of destruction seen on CT of the thorax. Other possibilities could be secondary to his HIV affecting his lungs; however, the patient was started on HAART therapy a few months earlier with appropriate response in both CD4 count and viral load, so that concern had already been addressed. Alpha-1 antitrypsin deficiency could also explain the symptoms; however, this was ruled out. Therefore, what remained was the patient's pulmonary amyloidosis, which is known to cause an obstructive pattern on PFTs secondary to narrowing of the airways [2] and the evidence of cystic changes on thoracic CT, which are apparent in this patient. However, it is important to note the man's history of pneumocystic pneumonia, which might have led to cystic changes in the lungs, however these usually are limited in number, resolve with time and are not associated with severe obstructive lung disease [6].

This report is unique as the patient is the first described in literature to have both amyloid-related cystic lung disease and HIV. More case reports will need to be published and reviewed to see whether an association between HIV and amyloid-associated cystic lung disease exists.

\section{Conflict of interest}

None declared.

\section{References:}

1. Kyle RA. Amyloidosis: a convoluted story. Br J Haematol. 2001; 114(3): 529-538, doi: 10.1046/j.1365-2141.2001.02999.x, indexed in Pubmed: 11552976.

2. Zamora AC, White DB, Sykes AMG, et al. Amyloid-associated cystic lung disease. Chest. 2016; 149(5): 1223-1233, doi: 10.1378/chest.15-1539, indexed in Pubmed: 26513525.

3. Pepys MB. Amyloidosis. Annu Rev Med. 2006; 57: 223-241, doi: 10.1146/annurev.med.57.121304.131243, indexed in Pubmed: 16409147.

4. Gillmore JD, Hawkins PN. Amyloidosis and the respiratory tract. Thorax. 1999; 54(5): 444-451, doi: 10.1136/thx.54.5.444, indexed in Pubmed: 10212113.

5. Colombat M, Caudroy S, Lagonotte E, et al. Pathomechanisms of cyst formation in pulmonary light chain deposition disease. Eur Respir J. 2008; 32(5): 1399-1403, doi: 10.1183/09031936.00132007, indexed in Pubmed: 18978139.

6. Ryu JH, Tian X, Baqir M, et al. Diffuse cystic lung diseases. Front Med. 2013; 7(3): 316-327, doi: 10.1007/s11684-0130269-z, indexed in Pubmed: 23666611. 